\section{EFEITO DA ORDEM DOS EXERCÍCIOS DE FORÇA SOBRE O VOLUME, O LACTATO E O DESEMPENHO}

\author{
EFFECT OF THE ORDER OF STRENGTH EXERCISES ON VOLUME, LACTATE AND PERFORMANCE
}

EFECTO DE LA ORDEN DELOS EJERCICIOS DE FUERZA EN EL VOLUMEN, LACTATO YRENDIMIENTO

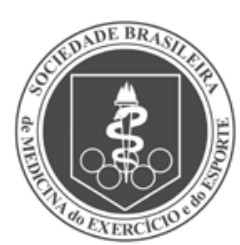

Artigo Original

Original Article Artículo Original
Lucas Duarte Tavares'

(Profissional de Educação Física)

Carlos Ugrinowitsch ${ }^{1}$

(Profissional de Educação Física)

Cláudio Machado Pinto e Silva ${ }^{1}$

(Profissional de Educação Física)

Eduardo Oliveira de Souza 1,2

(Profissional de Educação Física)

Gilberto Cândido Laurentino'

(Profissional de Educação Física)

Fabiano Aparecido Pinheiro'

(Profissional de Educação Física)

Thiago Lasevicius $^{1}$

(Profissional de Educação Física)

Valmor Tricoli ${ }^{1}$

(Profissional de Educação Física)

1. Universidade de São Paulo, Escola de Educação Física e Esporte, Laboratório de Adaptação ao Treinamento de Força, São Paulo, SP, Brasil.

2. University of Tampa, Department of Health Sciences and Human Performance, Tampa, FL, USA.

\section{Correspondência:}

Valmor Tricoli

Departamento de Esporte. Escola de Educação Física e Esporte. Universidade de São Paulo. Av. Prof. Mello Moraes, 65, Butantã, São Paulo, SP, Brasil. 05508-030. vtricoli@usp.br

\section{RESUMO}

Introdução: A alternância entre segmentos corporais para prescrição do treinamento de força (TF) é amplamente utilizada, entretanto seus efeitos permanecem desconhecidos. Objetivo: Verificar o efeito agudo da manipulação da ordem dos exercícios do TF, alternando segmentos corporais, sobre a força dinâmica máxima (1 RM) de membros inferiores (MMII) e superiores (MMSS), a concentração de lactato sanguíneo [La], a percepção subjetiva de esforço (PSE) e o volume total de treino (VT). Métodos: Dezoito homens ( $23,1 \pm 3,8$ anos; $78,4 \pm$ 8,6 kg; 1,72 $\pm 0,06$ m) executaram três condições de TF: condição A (supino reto, desenvolvimento, rosca Scott, meio-agachamento, leg press, cadeira extensora); condição B (meio-agachamento, leg press, cadeira extensora, supino reto, desenvolvimento, rosca Scott) e condição C (supino reto, meio-agachamento, desenvolvimento, leg press, rosca Scott e cadeira extensora). Os exercícios foram executados com três séries de 10 RM. Os testes de 1 RM para MMII e MMSS foram realizados nas semanas iniciais e após as condições experimentais. As coletas de sangue foram feitas no repouso [T0], imediatamente após [IA], 3 [T3] e 5 minutos [T5] após cada condição. A PSE de MMSS, MMIl e geral, e o VT foram mensurados ao final de cada condição. Resultados: A 1 RM foi reduzida depois da sessão em todas as condições tanto para MMSS ( $A=-19,4 \% ; B=-23,0 \% ; C=-22,8 \%)$ quanto para MMII ( $A=-16,2 \% ; B=-15,3 \% ; C=-16,1 \%)$ sem diferença entre condições. A PSE apresentou resultados similares entre as condições (Condição $A=8,4$; $C$ ndição $B=8,7$; Condição $C=8,7$ ). Houve aumento significante no La


$B=6,51$ mmol. $\left.\mathrm{L}^{-1}\right)(\mathrm{p}<0,0001)$. O VT na condição $C(16.169,4 \pm 2.562,5 \mathrm{~kg})$ foi superior às condições $A(13.222,2 \pm$ $2.010,4 \mathrm{~kg})$ e $B(13.989,8 \pm 2.223,4 \mathrm{~kg})(\mathrm{p}<0,0001)$. Conclusão: Os resultados sugerem que somente o VT e a demanda metabólica foram mais afetados pela ordem dos exercícios.

Descritores: fadiga; ácido láctico; tolerância ao exercício.

\section{ABSTRACT}

Introduction: The alternation between body segments for prescription of strength training (ST) is widely used; however, its effects remain unknown. Objective: To verify the acute effect of the manipulation of the order of ST exercises, alternating body segments, on the maximum dynamic force (1 RM) of lower limbs (LL) and upper limbs (UL), blood lactate [La] concentration, subjective perception of effort (SPE) and the total training volume (TV). Methods: Eighteen men (23.1 \pm 3.8 years; $78.4 \pm 8.6 \mathrm{~kg} ; 1.72 \pm 0.06 \mathrm{~m}$ ) performed three ST conditions: condition A: (bench press, shoulder press, preacher arm curl, half squat, leg press, leg extension); condition B (half squat, leg press, leg extension, bench press, shoulder press, preacher arm curl) and condition C (bench press, half squat, shoulder press, leg press, preacher arm curl, leg extension). The exercises were performed in three sets of 10 RM. The 1 RM tests for $L L$ and UL were performed in the initial weeks and after the experimental conditions. Blood samples were taken at rest [TO], immediately after [IA], 3 [T3], and 5 minutes [T5] after each condition. SPE of UL, LL, and general, and VT were measured at the end of each condition. Results: The 1 $R M$ was reduced after the session in all conditions for both $U L(A=-19.4 \%, B=-23.0 \%, C=-22.8 \%)$ and $L L(A=-16.2 \%$, $B=-15.3 \%, C=-16.1 \%)$ with no difference between conditions. The SPE presented similar results between the conditions (Condition $A=8.4$, Condition $B=8.7$, Condition $C=8.7$ ). There was a significant increase in La at time $T 3$ of condition $C$ compared to the other conditions: $\left(C=7.29 \mathrm{mmol}^{-L^{-1}}, A=6.60 \mathrm{mmol} . \mathrm{L}^{-1}, B=6.51 \mathrm{mmol} . \mathrm{L}^{-1}\right)(p<0.0001)$. The TV in condition $C(16,169.4 \pm 2,562.5 \mathrm{~kg})$ was higher than conditions $A(13,222.2 \pm 2,010.4 \mathrm{~kg})$ and $B(13,989.8 \pm 2223.4 \mathrm{~kg})(p<0.0001)$. Conclusion: The results suggest that only TV and the metabolic demand were more affected by the order of exercises.

Keywords: fatigue; lactic acid; exercise tolerance.

\section{RESUMEN}

Introducción: La alternancia entre los segmentos del cuerpo para la prescripción de entrenamiento de fuerza (EF) es ampliamente utilizada, pero siguen desconocidos sus efectos. Objetivo: Evaluar el efecto agudo de la manipulación de la orden de los ejercicios de EF, alternando segmentos del cuerpo, en la fuerza máxima (1 RM) de las extremidades inferiores (EI) y superiores (ES), la concentración del lactato (La) en la sangre, la percepción subjetiva del esfuerzo (PSE) y el volumen de entrenamiento (VE). Métodos: Dieciocho hombres (23,1 $\pm 3,8$ años; $78,4 \pm 8,6 \mathrm{~kg} ; 1,72 \pm 0,06 \mathrm{~m})$ realizaron tres condiciones de EF: Condición A (press de banca, press de hombros, rosca Scott, media sentadilla, prensa de piernas, extensión de la pierna); Condición B (media sentadilla, prensa de piernas, extensión de la pierna, press de banca, press de ombros, rosca Scott) y Condición C (press de banca, media sentadilla, press de hombros, prensa de piernas, rosca Scott, 
y extensión de la pierna). Los ejercicios se realizaron con tres series de 10 RM. Las pruebas de 1 RM de las Ely ES sellevaron a cabo en las primeras semanas y después de las condiciones experimentales. Las muestras de sangre fueron tomadas en reposo [TO] inmediatamente después [IA], 3 [T3] y 5 minutos [T5] después de cada condición. La PSE, de ES, Ely general y VE se midieron al final de cada condición. Resultados: La 1 RM se redujo después de la sesión en todas las condiciones para ES $(A=-19,4 \% ; B=-23,0 \% ; C=-22,8 \%)$ y El $(A=-16,2 \% ; B=-15,3 \% ; C=-16,1 \%)$, sin diferencia entre las condiciones. La PSE mostró resultados similares entre las condiciones (Condición $A=8,4$, Condición $B=8.7$, Condición $C=8,7$ ). Hubo un aumento significativo en $L a$ en el momento T3 de la condición Cen comparación con las otras condiciones ( $C=7,29$ mmol. $\left.L^{-1} ; A=6,60 \mathrm{mmol} . L^{-1}, B=6,51 \mathrm{mmol} . \mathrm{L}^{-1}\right)(p<0,0001)$. El VEen la condición $C(16.169,4 \pm 2.562,5 \mathrm{~kg})$ fue superior a las condiciones $A(13.222,2 \pm 2.010 .4 \mathrm{~kg})$ y $B(13.989,8 \pm 2.223,4 \mathrm{~kg})(p<0,0001)$. Conclusión: Los resultados sugieren que solamente el VEy las demandas metabólicas fueron afectados por el orden de los ejercicios.

Descriptores: fatiga; ácido láctico; tolerancia al ejercicio.

\section{INTRODUÇÃO}

A manipulação das variáveis envolvidas no treinamento de força (TF) como o volume, a intensidade, o intervalo de descanso entre as séries e a ordem dos exercícios tem sido realizada com o intuito de otimizar as adaptações produzidas pelo TF no que se refere ao aumento da força e da massa muscular ${ }^{1,2}$.

Especificamente, a manipulação da ordem dos exercícios em sessões de TF e seu efeito sobre diferentes variáveis de desempenho e sobre o estresse metabólico tem despertado interesse da comunidade científica ${ }^{3-5}$. Neste sentido, alguns estudos agudos têm investigado como a sequência de exercícios partindo de grandes grupos musculares (i.e. exercícios multiarticulares ou grupos musculares de maior tamanho) para pequenos grupos musculares (i.e. exercícios monoarticulares ou grupos musculares de menor tamanho) ou vice-versa pode afetar a demanda metabólica (e.g. concentrações de lactato sanguíneo [La]), a percepção subjetiva de esforço (PSE) e o desempenho de diferentes manifestações da força muscular (e.g. força máxima e/ou resistência de força $)^{3-10}$. Sabe-se que a manipulação da ordem dos exercícios possui associação direta com a magnitude do intervalo de descanso entre séries e exercícios, e com o volume total (VT) de uma sessão de treino ${ }^{11,12}$. Neste contexto, alguns investigadores têm observado que sessões de TF iniciadas por exercícios multiarticulares resultam em maiores VT, e consequentemente, em aumentos mais pronunciados nas [La] sanguíneo, o que pode afetar o desempenho da força muscular e o comportamento da PSE ${ }^{1,3-5}$. No entanto, até o momento os estudos têm manipulado a ordem dos exercícios isolando segmentos corporais específicos (i.e. a realização de todos os exercícios de membros superiores para posteriormente realizar os exercícios de membros inferiores ou vice-versa).

Poucos estudos ${ }^{13,14}$ verificaram o efeito da alternância dos exercícios envolvendo os diferentes segmentos corporais (membros superiores e inferiores), sendo que ainda permanecem pouco esclarecidos na literatura os efeitos dessa estratégia de realização do TF, no volume total de treinamento (VT), na demanda metabólica (e.g. [La]), e no desempenho da força dinâmica máxima (i.e. 1RM). A alternância dos segmentos corporais pode promover maiores intervalos de recuperação intra-segmento muscular durante a realização da sessão de treino, o que em tese, poderia auxiliar a prevenir a ocorrência de fadiga precoce em comparação às outras estratégias de manipulação da ordem dos exercícios. Desta forma, é possível que a manipulação da ordem dos exercícios a partir da alternância dos segmentos corporais possibilite aumentar a quantidade de carga utilizada em cada exercício durante uma sessão de treinamento. Diante dessa premissa, a alternância dos segmentos corporais poderia favorecer a produção de maiores VT na sessão de TF, e consequentemente promover aumentos na [La], conjuntamente com as respostas da PSE sem apresentar prejuízos no desempenho da força dinâmica máxima ${ }^{15-18}$.

Portanto, o objetivo do presente estudo foi verificar o efeito agudo da manipulação da ordem dos exercícios do TF, alternada por segmentos corporais, no desempenho da força dinâmica máxima, na PSE, na [La] sanguíneo e no VT em indivíduos fisicamente ativos e sem experiência prévia em TF.

\section{MATERIAIS E MÉTODOS}

O estudo foi conduzido por um período de seis semanas (Figura 1). Nas duas primeiras semanas, todos os sujeitos foram submetidos a três sessões de familiarização ao teste de força dinâmica máxima (1RM) para membros inferiores e superiores com 48 horas de intervalo entre as sessões, tendo o teste propriamente dito, sido efetuado após a terceira sessão de familiarização (pré-teste). Na terceira semana, os sujeitos participaram de duas sessões (separadas por 48-72 horas) de teste de repetições máximas (10 RM) para familiarização aos exercícios de treinamento e para a determinação das cargas a serem utilizadas nos protocolos experimentais. Nas semanas seguintes (4-6), todos os sujeitos executaram as três condições experimentais (desenho crossover), as quais foram distribuídas de maneira aleatória e randomizada pelo procedimento dos quadrados latinos ${ }^{19}$ e identificadas no Quadro 1. Imediatamente ao final de cada condição experimental, os sujeitos foram submetidos aos testes de 1RM para os membros inferiores (MMII) e superiores (MMSS). Além disso, foi realizada a avaliação da PSE de MMSS, MMII e a percepção geral da sessão e coleta de sangue para medida da concentração de lactato no repouso (T0), imediatamente após (IA), 3 minutos (T3) e 5 minutos (T5) após a realização das condições.

\section{Amostra}

O tamanho da amostra foi determinado a partir de um estudo piloto previamente realizado, utilizando indivíduos que possuíam as mesmas características daquelas que foram empregadas no presente estudo, baseando-se em uma significância de 5\% e um poder do teste

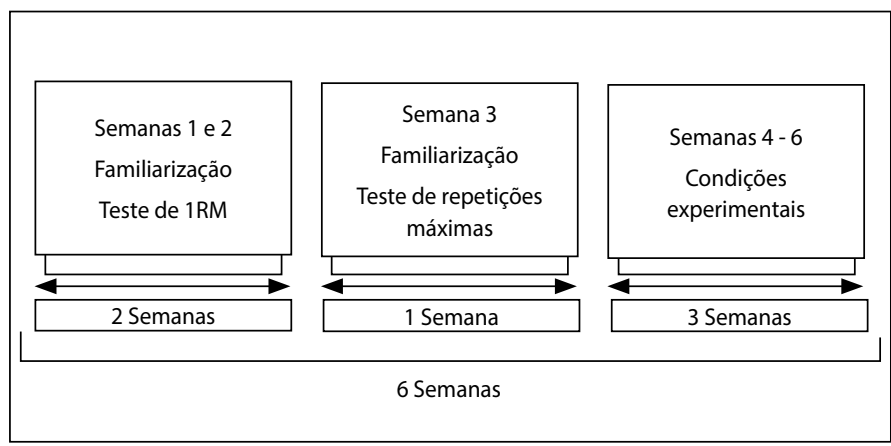

Figura 1. Distribuição temporal dos procedimentos experimentais do estudo. 
Quadro 1. Ordem dos exercícios nas condições experimentais do estudo.

\begin{tabular}{|c|c|c|c|}
\hline $\begin{array}{c}\text { Ordem dos } \\
\text { exercícios }\end{array}$ & Condição A & Condição B & Condição C \\
\hline $1^{\circ}$ & Supino reto & Meio-agachamento & Meio-agachamento \\
\hline $2^{\circ}$ & $\begin{array}{c}\text { Desenvolvimento } \\
\text { frontal }\end{array}$ & Cadeira extensora & Supino reto \\
\hline $3^{\circ}$ & Rosca Scott & Leg press & Cadeira extensora \\
\hline $4^{\circ}$ & Meio-agachamento & Supino reto & $\begin{array}{c}\text { Desenvolvimento } \\
\text { frontal }\end{array}$ \\
\hline $5^{\circ}$ & Cadeira extensora & $\begin{array}{c}\text { Desenvolvimento } \\
\text { frontal }\end{array}$ & Leg press \\
\hline $6^{\circ}$ & Leg press & Rosca Scott & Rosca Scott \\
\hline
\end{tabular}

de $80 \%{ }^{19}$. Participaram do estudo 18 sujeitos do sexo masculino (idade $23,1 \pm 3,8$ anos, massa corporal 78,4 $\pm 8,6 \mathrm{~kg}$ e estatura 1,72 $\pm 0,06 \mathrm{~m}$ ), fisicamente ativos, praticantes de atividades esportivas em nível recreativo com frequência de duas sessões por semana, no mínimo por dois anos e sem experiência prévia com TF. Os sujeitos não apresentavam qualquer problema neuromuscular ou ortopédico nos membros inferiores e superiores nos seis meses que precederam o estudo. Todos os participantes receberam informações sobre os riscos e benefícios do estudo e assinaram um termo de consentimento informado antes da participação voluntária. O estudo foi aprovado pelo comitê de ética local (Protocolo 2010/52).

\section{Teste de força dinâmica máxima (1RM)}

O desempenho da força dinâmica máxima de MMSS e MMII foi mensurado nos exercícios supino reto e meio-agachamento; respectivamente, no aparelho Smith Machine (Nakagym ${ }^{\circledR}$, Diadema, São Paulo, Brasil) antes e após a realização das condições experimentais. Para a realização dos testes de 1RM foram seguidas as orientações da Sociedade Americana de Fisiologistas do Exercício ${ }^{20}$. Antes do início do teste, os sujeitos executaram um aquecimento geral de cinco minutos pedalando em bicicleta ergométrica (Movement ${ }^{\circledR}$, São Paulo, Brasil) na velocidade de 70 rpm. O aquecimento específico consistiu em duas séries do próprio exercício, sendo que na primeira série, os sujeitos executaram oito repetições com uma carga estimada de 50\% 1RM e na segunda série três repetições com carga estimada de 70\% 1RM com intervalos de dois minutos entre as séries. Após um intervalo de três minutos o teste máximo foi iniciado. A carga inicial utilizada no teste de 1RM foi baseada nos valores obtidos durante as sessões de familiarização. Os incrementos de carga a cada tentativa foram de aproximadamente 2,5\% a 5\% da carga anterior. Para que a tentativa fosse considerada válida, os sujeitos deveriam executar um ciclo completo e correto de movimento no exercício específico. A execução do meio-agachamento foi feita com a barra apoiada sobre os ombros, e a posição inicial e final do movimento deveria ser com os joelhos estendidos com a posição intermediária correspondente a um ângulo de $90^{\circ}$ nos joelhos. Para o controle da amplitude, foram posicionados atrás dos sujeitos caixotes com altura regulável. Na execução do exercício supino reto, o ciclo do movimento teve início com os cotovelos estendidos, posteriormente a barra descia até tocar a região torácica e o movimento era finalizado com a extensão completa dos cotovelos. Foi determinado o máximo de cinco tentativas para alcançar o valor de 1RM com intervalo de 3-5 minutos entre cada tentativa. Durante todas as tentativas foi oferecido encorajamento verbal aos sujeitos. Os mesmos procedimentos, com exceção dos aquecimentos geral e específico, foram seguidos nos testes ao final de cada condição experimental.

\section{Teste de repetições máximas (10 RM)}

O teste de 10 RM foi feito na seguinte ordem: meio-agachamento, supino reto, leg-press, desenvolvimento frontal, cadeira extensora e rosca Scott. Os exercícios foram selecionados devido à sua ampla utilização em centros de treinamento e facilidade de execução. As instruções padronizadas foram fornecidas antes do teste, de modo que o avaliado estivesse ciente de toda a rotina que envolvia a coleta de dados. Foram aplicadas três tentativas, com intervalos entre as tentativas fixados entre três e cinco minutos. Após obtenção da carga em um determinado exercício, intervalos não inferiores a cinco minutos foram dados, antes de passar ao teste no exercício seguinte. Foram definidos como parâmetros de execução dos exercícios: posição inicial e a realização das fases concêntrica e excêntrica da contração. Os exercícios supino reto e meio-agachamento seguiram as mesmas recomendações aplicadas para o teste de 1RM. Nos demais exercícios, os parâmetros de realização do exercício são descritos a seguir: 1) leg press: inicialmente, o apoio para os pés no equipamento foi dividido em quadrados de $10 \times 10 \mathrm{~cm}$ com fita adesiva permitindo a anotação do posicionamento dos pés numa folha de papel e garantindo a reprodução do mesmo em todas as sessões de testes. Em seguida, a amplitude de movimento para cada repetição foi determinada. Os sujeitos realizaram uma repetição sem carga, começando com a extensão completa dos joelhos até $90^{\circ}$ de flexão e um goniômetro foi utilizado para confirmar o grau de flexão estipulado. Em seguida, um marcador de plástico foi colocado sobre a coluna lateral do equipamento leg press $45^{\circ}$ para demarcar o ponto de flexão do joelho. Uma fita de medição também foi colada sobre a coluna lateral do aparelho para assegurar a reprodutibilidade do posicionamento do marcador; 2) desenvolvimento frontal: inicialmente, o sujeito era orientado a ficar sentado no banco vertical com o tronco totalmente apoiado, joelhos semiflexionados, com os cotovelos estendidos e braços elevados. A realização do exercício iniciava a partir da flexão de $90^{\circ}$ entre braço e antebraço, seguida da extensão completa dos cotovelos com abdução de ombros; 3) rosca Scott: inicialmente, o indivíduo foi orientado a ficar em pé, mantendo os pés paralelos com pequeno afastamento lateral, com os joelhos semiflexionados, quadris na posição anatômica, cotovelos estendidos, com as mãos voltadas para frente segurando a barra, e a cabeça posicionada no plano de Frankfurt; foi realizada a flexão completa dos cotovelos a partir de $180^{\circ}$ entre braço e antebraço; 4) cadeira extensora: inicialmente, os sujeitos eram orientados a ficar sentados, com o tronco totalmente apoiado na cadeira extensora. O ciclo de movimento iniciou-se com os joelhos em um ângulo de $90^{\circ}$ e ao sinal do avaliador o sujeito realizava a fase concêntrica do movimento estendendo os joelhos até alcançar o ângulo de $180^{\circ} \mathrm{e}$ depois os flexionava novamente até a posição inicial. Após a obtenção das cargas máximas no teste de 10 RM, os indivíduos descansaram por 48 horas e foram reavaliados para obtenção da reprodutibilidade do teste (teste e reteste). Considerou-se como 10RM a carga estabelecida em ambos os dias com diferença menor que 5\%. Nos intervalos entre as sessões de testes não foi permitida a realização de exercícios para não interferir nos resultados obtidos. As cargas usadas nas condições experimentais foram as maiores obtidas durante a realização dos testes, sendo sua reprodutibilidade verificada através do teste de correlação intraclasse (ICC) (meio-agachamento $R=0,98$; supino reto $R=0,99$; leg press $R=1,00$; desenvolvimento frontal $R=0,97$; cadeira extensora $R=0,98$; rosca Scott $R=0,99$ ).

\section{Percepção subjetiva de esforço (PSE)}

A PSE de cada condição experimental foi avaliada com o uso da escala de Borg CR-1021. Nessa escala, o valor máximo (10) deveria ser referente ao maior esforço físico experimentado pelo sujeito e o valor 
mínimo (0) relativo à condição de repouso absoluto. Antes do início e imediatamente após a realização das condições experimentais, foi solicitado ao indivíduo que identificasse na escala de 0 a 10 sua percepção de esforço geral (i.e., corpo todo) e para os diferentes segmentos corporais (i.e, membros inferiores ou membros superiores) envolvidos nos exercícios da sessão de treinamento.

A medida da concentração de lactato sanguíneo [La] foi realizada em cada condição experimental. Para tanto, foram coletadas amostras de sangue de $25 \mu \mathrm{l}$ no lóbulo da orelha e analisadas no aparelho Yellow Springs 1500 Sports (YSI, Yellow Springs, Ohio, EUA). As amostras foram imediatamente alojadas em microtubos de polietileno do tipo Eppendorff, com capacidade para até $1,5 \mathrm{~mL}$, e armazenadas a $-70^{\circ} \mathrm{C}$, para posterior dosagem. Cada microtubo continha $50 \mu \mathrm{l}$ de fluoreto de sódio 1\%. As coletas foram efetuadas nas situações de repouso (T0), imediatamente após (IA), três (T3) e cinco minutos (T5) após o término das condições experimentais.

\section{Volume total da sessão de treinamento (VT)}

OVT para os MMSS, MMIl e o geral (MMSS + MMII) foram calculados multiplicando-se o número de séries dos exercícios pelo número de repetições e a carga utilizada (kg) nas diferentes condições experimentais. Para os MMSS foram utilizados os exercícios supino reto, desenvolvimento frontal e rosca Scott e para os MMII os exercícios meio-agachamento, leg press e cadeira extensora.

Nas diferentes condições experimentais foram realizadas três séries de 10 repetições máximas com as cargas determinadas no teste de repetições máximas em cada exercício. As cargas utilizadas foram ajustadas ao longo dos exercícios, com base na percepção do executante e de um avaliador experiente, para a manutenção do número de repetições pré-determinado. Foi dado um intervalo de dois minutos entre as séries e entre os exercícios. Na condição A, foram efetuados inicialmente os exercícios para MMSS (supino reto, desenvolvimento frontal, rosca Scott) e posteriormente os exercícios para MMII (meio-agachamento, cadeira extensora, leg press). Na condição B, a ordem proposta foi inversa, sendo efetuados primeiramente os exercícios de MMII e posteriormente os exercícios de MMSS. Por fim na condição C, os exercícios foram efetuados de maneira alternada, sendo realizado um exercício de MMII e posteriormente um exercício para MMSS (Quadro 1).

\section{Análise estatística}

A normalidade dos dados foi verificada com a aplicação do teste de Shapiro Wilk. Uma vez que os dados de desempenho da força, das concentrações de lactato, do volume total e da PSE apresentaram distribuição normal, a estatística paramétrica foi utilizada. Uma ANOVA one way assumindo as condições experimentais ( $A$, B e C) como fator foi utilizada para verificar o efeito da ordem dos exercícios sobre o volume total de treinamento e a PSE de MMII, de MMSS e geral. Uma ANOVA (3 $\times 2)$ para medidas repetidas, assumindo as condições experimentais ( $A$, $B$ e C) e tempo (antes e após condição experimental) como fatores foi utilizada para verificar o efeito da ordem dos exercícios sobre o desempenho da 1RM. Uma ANOVA $(3 \times 4)$ para medidas repetidas assumindo as condições experimentais ( $\mathrm{A}$, B e C) e tempo (T0, IA, T3 e T5) como fatores foi utilizada para verificar o efeito da ordem dos exercícios sobre a concentração de lactato sanguíneo. Na presença de F significante, o teste post-hoc de Tukey foi utilizado para identificar as diferenças. O nível de significância foi estabelecido em $p \leq 0,05$. Os dados são apresentados com média \pm desvio padrão.

\section{RESULTADOS}

Com relação ao desempenho no teste de 1RM, houve diminuição significante na força dinâmica máxima dos membros inferiores e superiores em todas as condições experimentais após a execução dos protocolos de exercícios. Foram observadas quedas similares entre as condições experimentais nos exercícios meio-agachamento (Condição $A=-16,2 \%$; Condição $B=-15,3 \%$; Condição $C=-16,1 \%$ ) e supino reto (Condição $A=-19,4 \%$; Condição $B=-23,0 \%$; Condição $C=-22,8 \%)(p<0,001)$. Em adição, não houve diferenças significantes entre as condições experimentais ( $p>0,05)$ (Tabela 1).

Não houve diferença significante nos valores de PSE geral, PSE MMII e PSE MMSS entre as condições experimentais $(p>0,05)$. Contudo, deve ser destacado que os valores de PSE no início das condições experimentais foram iguais ao valor de repouso absoluto, e os valores encontrados após a execução das condições indicaram um nível de esforço elevado segundo a classificação da escala de Borg CR-10 (Tabela 2).

Houve um aumento significante nas concentrações de lactato em todas as condições experimentais quando comparadas ao $T 0$ ( $F=20,25 ; p<0,05)$. Os aumentos nas [La] nos momentos T3 eT5 foram superiores quando foram comparados ao momento IA ( $F=46,95 ; \mathrm{p}<0,05)$; no entanto, não houve diferença significante entre esses momentos $(p>0,05)$. Adicionalmente, 0 aumento na concentração de lactato na condição C foi significantemente superior às demais condições no momento T3 ( $F=0,49 ; p<0,0001$ ) (Tabela 3).

Em relação ao VT, após a realização dos exercícios, a condição C apresentou valores maiores em comparação as demais condições no VT de MMSS e MMII ( $F=3,97 ; p<0,005)$. Da mesma forma, foi observado que o VT geral (MMII + MMSS) foi significantemente maior na condição C, em comparação as outras condições ( $F=4,17 ; p<0,0001)$. Nas demais condições, os valores de VT dos MMSS foram maiores na condição A em comparação com a condição $B(F=4,53 ; p<0,005)$. Os valores de VT dos MMll foram maiores na condição B em comparação a condição $A(F=4,96 ; p<0,005)$ (Figura 2).

Tabela 1. Desempenho da força dinâmica máxima (1RM, kg) nos exercícios meio-agachamento e supino reto, antes e após as condições experimentais (média \pm DP).

\begin{tabular}{c|c|c|c|c}
\hline Exercício & Pré & $\begin{array}{c}\text { Pós-condição } \\
\text { A }\end{array}$ & $\begin{array}{c}\text { Pós-condição } \\
\text { B }\end{array}$ & $\begin{array}{c}\text { Pós-condição } \\
\text { C }\end{array}$ \\
\hline Meio-agachamento & $163,4 \pm 27,2$ & $136,9 \pm 30,7^{*}$ & $138,3 \pm 29,8^{*}$ & $137,0 \pm 29,2^{*}$ \\
\hline Supino reto & $85,9 \pm 18,4$ & $69,2 \pm 18,7^{*}$ & $66,1 \pm 16,8^{*}$ & $66,3 \pm 16,8^{*}$ \\
\hline
\end{tabular}

Tabela 2. Percepção subjetiva de esforço (U.A.) geral e para os membros inferiores e superiores após as condições experimentais (média \pm DP).

\begin{tabular}{c|c|c|c}
\hline & Condição A & Condição B & Condição C \\
\hline PSE geral & $8,4 \pm 1,2$ & $8,7 \pm 0,9$ & $8,7 \pm 1,3$ \\
\hline PSE MMII & $9,1 \pm 0,8$ & $8,7 \pm 0,9$ & $8,6 \pm 1,0$ \\
\hline PSE MMSS & $8,6 \pm 0,8$ & $9,2 \pm 0,8$ & $9,1 \pm 0,8$ \\
\hline
\end{tabular}

PSE geral = percepção do esforço em relação ao corpo todo. PSE MMII = percepção do esforço associada à reposta de membros inferiores. PSE MMSS = percepção do esforço associada à resposta de membros superiores.

Tabela 3. Concentrações de lactato sanguíneo [La] (mmol. $\mathrm{L}^{-1}$ ) antes (T0), imediatamente após (IA), 3 minutos (T3) e 5 minutos após (T5) as condições experimentais (média \pm DP).

\begin{tabular}{c|c|c|c}
\hline & Condição A & Condição B & Condição C \\
\hline T0 & $0,63 \pm 0,15$ & $0,68 \pm 0,19$ & $0,63 \pm 0,20$ \\
\hline IA & $5,8 \pm 1,32^{*}$ & $5,71 \pm 1,28^{*}$ & $5,98 \pm 1,84^{*}$ \\
\hline T3 & $6,6 \pm 1,05^{*+}$ & $6,51 \pm 1,15^{*+}$ & $7,29 \pm 1,74^{*}+\$$ \\
\hline T5 & $6,13 \pm 1,37^{*+}$ & $5,93 \pm 1,08^{*+}$ & $6,27 \pm 2,02^{*}+$ \\
\hline
\end{tabular}

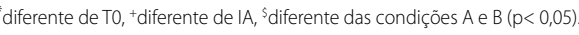

\section{DISCUSSÃO}

O objetivo do presente estudo foi verificar o efeito agudo da manipulação da ordem dos exercícios do TF, alternada por segmentos corporais, no desempenho da 1RM, na PSE, na [La] sanguíneo e noVT em indivíduos fisicamente ativos e sem experiência prévia em TF. Os principais achados foram: 




Figura 2. Volume total de treinamento (kg) para os membros inferiores (MMII), membros superiores (MMSS) e para o corpo todo (MMII + MMSS) nas diferentes condições experimentais (média \pm DP).

1) a $1 R M$ apresentou reduções significantes e semelhantes entre as diferentes condições de TF; 2) os valores da PSE não apresentaram diferenças significantes entre as três condições experimentais; 3) a [La] sanguíneo apresentou aumentos similares; contudo, no momento T3 da condição C a concentração foi maior quando comparada ao mesmo período das condições $A$ e B; 4) na condição em que os segmentos corporais foram alternados (condição C), o volume de treino (VT) foi significantemente maior em comparação às demais condições.

Em relação ao desempenho da força dinâmica máxima, foram observadas diminuições significantes da força de MMII e MMSS ( 18-20\% e 25-30\%, respectivamente) após as condições experimentais. De maneira similar, Simão et al. ${ }^{8}$ verificaram queda de $25 \%$ no desempenho da força de MMII no exercício leg press após a realização de protocolos de TF com diferentes ordens dos exercícios (multiarticulares-monoarticulares ou monoarticulares-multiarticulares). Da mesma forma foi observado no estudo de Gil et al. ${ }^{18}$ diminuição de aproximadamente $30 \%$ no desempenho da força de MMII no exercício leg press quando o teste foi realizado no final da sessão de TF após os exercícios cadeira flexora e cadeira extensora. Outro estudo de Simão et al. ${ }^{22}$ também demonstrou diminuição de aproximadamente 30\% no desempenho da força de MMSS e MMII durante após a realização de uma sessão de TF com diferentes ordens (multiarticulares ou monoarticulares), mesmo com intensidade mais baixa (i.e., 20 RM) do que a utilizada no presente estudo. Entretanto, é importante ressaltar que no presente estudo o desempenho da força foi mensurado através do teste de 1RM, enquanto nos estudos destacados, o desempenho medido através do teste de repetições máximas. Além disso, nos estudos mencionados, a manipulação da ordem dos exercícios foi efetuada em função do número de articulações envolvidas nos exercícios e específicas para um segmento corporal.

Somente o estudo de Romano et al. ${ }^{13}$ verificou o efeito da ordem dos exercícios alternada por segmentos corporais (Sequência $A=$ leg press, afundo, supino reto, tríceps pulley; Sequência B = leg press, supino, afundo, tríceps pulley). Estes autores observaram reduções de aproximadamente 20\% no desempenho da 1RM de MMII e MMSS. No presente estudo observamos que, independente da manipulação da ordem dos exercícios executados na sessão de TF, houve um decréscimo no desempenho de força, provavelmente em função de alterações nos mecanismos que modulam a produção da força máxima. Alguns estudos sugerem que a diminuição do desempenho da força esteja associada à mecanismos de ordem neural envolvidos na produção de força muscular (e.g. recrutamento de unidades motoras, ativação muscular, taxa de desenvolvimento de força), independentemente da ordem dos exercícios, do intervalo entre séries e exercícios, e do VT executado na sessão ${ }^{22-25}$. Para exemplificar, Marshaw et al..$^{23}$ observaram que em sessões de TF com diferentes volumes (i.e., 1x10, $4 \times 9$ e 8x8), a diminuição na ativação muscular foi similar ao término das sessões e sem diferenças entre as condições. Além da relação com o VT, o mesmo grupo de pesquisadores demonstrou em outro estudo ${ }^{24}$ que sessões de TF com diferentes intervalos entre séries (i.e., 20 segundos, 80 segundos e 720 segundos) apresentam diminuições equivalentes na força isométrica máxima. Embora o presente estudo não tenha mensurado os fatores anteriormente mencionados, foi possível verificar que, independentemente da alternância entre os segmentos corporais, houve uma diminuição similar da 1RM entre as condições experimentais, sugerindo que a intensidade utilizada nas sessões parece ser mais determinante na diminuição da força. Desta forma, pode-se afirmar que os fatores associados à capacidade do indivíduo produzir força sejam afetados igualmente, independentes da ordem dos exercícios utilizados na sessão de TF.

Com a relação à PSE, não foram observadas diferenças significantes entre as condições experimentais. Embora a PSE seja uma ferramenta frequentemente utilizada para monitoramento da intensidade relativa durante o TF, os estudos efetuados até o momento demonstram respostas similares na PSE, durante a realização de protocolos de TF com diferentes ordens de realização dos exercícios ${ }^{5-9}$. Por exemplo, Simão et al. ${ }^{8}$ verificaram que independentemente da sessão de TF iniciar com exercícios multi ou monoarticulares, a PSE apresentou alterações similares desde que a intensidade e o intervalo entre as séries também sejam similares. Em todas as condições experimentais efetuadas neste estudo, os sujeitos realizaram a mesma intensidade, quantidade de séries e repetições, tendo apenas alterações na sequência de realização dos exercícios. Embora na condição C tenha sido observado um maior VT, é possível que no presente estudo, os resultados da PSE estejam mais associados com a intensidade dos exercícios, o que parece justificar a similaridade observada entre as condições experimentais, bem como a diminuição similar observada também no desempenho da força máxima.

Paralelamente, a [La] sanguíneo tem sido usada como um indicador da resposta metabólica ao TF3,4,11,15,17,26,27. Os resultados obtidos no presente estudo estão parcialmente de acordo com os achados da literatura 3,4,26,27. No estudo efetuado por Smilios et al. ${ }^{26}$ foram observados aumentos significativos na [La] sanguíneo em condições que tinham como exercícios o leg press, o supino reto, a cadeira extensora e a rosca direta com intervalos iguais ou maiores que dois minutos com aproximadamente 15 repetições a $60 \%$ 1 RM. Em outro trabalho, Belleza et al. ${ }^{4}$ verificaram aumentos significantes nas [La] sanguíneo após a realização de uma sessão de TF em que eram efetuados os exercícios leg press, agachamento e supino reto com intervalos entre 2-4 minutos e com séries de 10 RM. Entretanto, diferentemente do presente trabalho, nos estudos citados, os exercícios foram efetuados sem alternância entre os segmentos corporais. Neste estudo, foram verificados, aumentos significantes na [La] sanguíneo imediatamente, três e cinco minutos após as condições experimentais. Entretanto, a condição $C$ apresentou aumento significantemente diferente no T3, em comparação as demais condições experimentais. O protocolo de TF aplicado teve o uso de intervalos entre séries, com duração de dois minutos, de forma similar a alguns estudos realizados anteriormente ${ }^{4,27}$. Contudo, especula-se que a alternância entre os segmentos corporais, a qual possibilitou maior VT durante a realização do treino, pode ter influenciado a resposta metabólica observada no presente estudo 16,17,22,26,27. Corroborando com as investigações mencionadas anteriormente, é possível que o comportamento da [La] sanguíneo possua maior dependência do VT. Além disso, o fato das repetições terem sido executadas até a falha concêntrica pode ter aumentada a demanda metabólica em comparação a situações em que oTF é efetuado com repetições submáximas ${ }^{11,16,17}$. Assim, é possível que o maior trabalho realizado (e.g. volume total) observado na condição C seja um dos principais fatores para uma resposta mais acentuada da [La] nessa condição experimental. 
No presente estudo foi observado que o VT foi maior na condição experimental C (Figura 2). Assim, pode-se sugerir que a estratégia de alternar os segmentos corporais tenha promovido uma melhor recuperação intra-segmento mantendo um maior desempenho em cada exercício ao longo da sessão e resultando num maiorVT. Adicionalmente, os menores valores do VT (MMII + MMSS) observado nas condições experimentais A e $B$ em que os exercícios foram efetuados sem a alternância entre os segmentos corporais, sugerem que o intervalo de descanso entre as séries e entre os exercícios (2 minutos), podem não ter sido suficientes, para os indivíduos fisicamente ativos sem experiência em TF, para preservar a carga utilizada em cada série ${ }^{6-11}$. Resultados semelhantes foram encontrados por Sforzo e Touey ${ }^{7}$ os quais verificaram reduções significantes no VT de MMII ( 45\%) e MMSS (58\%) em indivíduos fisicamente ativos e sem experiência em TF após a execução de sessões de TF com exercícios concentrados por segmento corporal e com intervalos de descanso entre séries com duração de dois minutos. Em estudo realizado por Ronnestad et al. ${ }^{28}$ foi observado que protocolos de TF com exercícios de MMII e MMSS (leg press, cadeira extensora, cadeira flexora, supino sentado, remada sentada, pulley costas, rosca direta e desenvolvimento frontal) realizados conjuntamente na mesma sessão, com intervalos de quatro minutos entre séries e exercícios, apresentaram VT mais elevados quando comparados com intervalos de um minuto. De maneira similar, Leite et al. ${ }^{17}$ verificaram que sessões de TF com exercícios de MMSS e MMII (supino reto, puxador dorsal, leg press, cadeira extensora e cadeira flexora), e intervalos entre 2-4 minutos, apresentaram maiores VT quando comparados às sessões de treino com os mesmo exercícios e intervalos inferiores a dois minutos, independente da intensidade utilizada nas sessões de TF (i.e 6RM ou 12RM).

Coletivamente, os dados apresentados anteriormente combinados aos nossos achados sugerem que não somente a alternância dos segmentos corporais, mas também o intervalo de descanso adotado entre a realização dos exercícios, podem afetar o VT. Desta forma, é possível que a alternância entre segmentos corporais, tenha produzido maior VT por promover maiores intervalos de recuperação entre os exercícios de um mesmo segmento (Condição $C=8$ minutos; Condição $A$ $=2$ minutos; Condição $B=2$ minutos). A produção de uma maior VT sugere que essa estratégia de organização do TF, de maneira crônica, possa promover adaptações mais pronunciadas (e.g. maior aumento da força e da hipertrofia muscular), uma vez que as evidências têm demonstrado que o VT é uma variável fundamental para a hipertrofia muscular induzida pelo TF ${ }^{16,17,28-30}$.

\section{CONCLUSÃO}

Em conclusão, a manipulação da ordem dos exercícios a partir da alternância de segmentos corporais promoveu maior VT, e apresentou valores mais elevados de [La] três minutos após o término da sessão, quando comparada com as outras ordens dos exercícios. Por outro lado, independentemente da ordem dos exercícios o desempenho da 1RM diminuiu e a PSE aumentou de maneira equivalente em todas as ordens dos exercícios adotadas no estudo. Desta forma, é possível sugerir que a organização de uma sessão de TF com alternância de segmentos corporais em diferentes exercícios parece ser uma estratégia interessante quando o objetivo for maximizar o VT. Possivelmente, os modelos de treino com este tipo de rotina seriam mais eficientes para produzir maiores cargas agudas de treino e possibilitar melhores adaptações de forma crônica para diferentes populações (e.g., iniciantes em TF e atletas de modalidades esportivas). Contudo, estudos futuros precisam ser realizados para avaliar se os efeitos agudos positivos observados no VT durante sessões de TF com séries de exercícios alternadas por segmento corporal refletirão em melhores adaptações crônicas induzidas pelo TF.

Todos os autores declararam não haver qualquer potencial conflito de interesses referente a este artigo.

CONTRIBUIÇÕES DOS AUTORES: Cada autor contribuiu individual e significativamente para o desenvolvimento do manuscrito. LDT (0000-0003- 0941-6224)* VT $(0000-0002-9014-087 X)^{*}$ e CU $(0000-0001-8547-419 X)^{*}$ foram os principais contribuintes na redação do manuscrito. EOS (0000-0002-9382-7407)*, CMPS (0000-00018585-9495)*, FAP (0000-0002-2894-2046)* e TL (0000-0002-4682-7287)* realizaram as coletas de dados, acompanharam os voluntários e reuniram dados obtidos para posterior análise. GCL (0000-0003-4225-7376)*, TL, VT, CU e LDT avaliaram os dados da análise estatística. LDT, CMPS, GCL, EOS e VT realizaram a pesquisa bibliográfica, a revisão do manuscrito e contribuíram com o conceito intelectual do estudo. *ORCID (Open Researcher and Contributor ID).

\section{REFERÊNCIAS}

1. American College of Sports Medicine. American College of Sports Medicine position stand. Progression models in resistance training for healthy adults. Med Sci Sports Exerc. 2009;41(3):687-708.

2. Kraemer WJ, Ratamess NA. Fundamentals of resistance training: progression and exercise prescription. Med Sci Sports Exerc. 2004;36(4):674-88.

3. Balsamo S, Tibana RA, Nascimento DC, Franz CB, Lyons S, Faigenbaum A, et al. Exercise order influences number of repetitions and lactate levels but not perceived exertion during resistance exercise in adolescents. Res Sports Med. 2013;21(4):293-304

4. Bellezza PA, Hall EE, Miller PC, Bixby WR. The influence of exercise order on blood lactate, perceptual, and affective responses. J Strength Cond Res. 2009;23(1):203-8.

5. Da Silva NSL, Monteiro WD, Farinatti PDV. Influence of exercise order on the number of repetitions and perceived exertion in young and older women. Rev Bras Med Esporte. 2009;15(3):219-23.

6. Gentil P, Oliveira E, de Araújo Rocha JúniorV, do Carmo J, Bottaro M. Effects of exercise order on upper-body muscle activation and exercise performance. J Strength Cond Res. 2007;21(4):1082-6.

7. Sforzo GA, Touey PR. Manipulating exercise order affects muscular performance during a resistance exercise training session. J Strength Cond Res 1996;10(1):20-24.

8. Simão R, Farinatti PT, Polito MD, Maior AS, FleckSJ. Influence of exercise order on the number of repetitions performed and perceived exertion during resistance exercises. J Strength Cond Res. 2005;19(1):152-6.

9. Simão R, Farinatti PT, Polito MD, Viveiros L, Fleck SJ. Influence of exercise order on the number of repetitions performed and perceived exertion during resistance exercise in women. J Strength Cond Res. 2007;21(1):23-8

10. Spreuwenberg LP, Kraemer WJ, Spiering BA, Volek JS, Hatfield DL, Silvestre R, et al. Influence of exercise order in a resistance-training exercise session. J Strength Cond Res. 2006;20(1):141-4.

11. Crewther B, Cronin J, Keogh J. Possible stimuli for strength and power adaptation: acute metabolic responses. Sports Med. 2006;36(1):65-78

12. Peterson MD, Pistilli E, Haff GG, Hoffman EP, Gordon PM. Progression of volume load and muscular adaptation during resistance exercise. Eur J Appl Physiol. 2011;111(6):1063-71.

13. Romano N, Vilaça-Alves J, Fernandes HM, Saavedra F, Paz G, Miranda H, et al. Effects of resistance exercise order on the number of repetitions performed to failure and perceived exertion in untrained young males. J Hum Kinet. 2013;39:177-83.

14. Ciccone AB, Brown LE, Coburn JW, Galpin AJ. Effects of traditional vs.alternating whole-body strength training on squat performance. J Strength Cond Res. 2014;28(9):2569-77.

15. Charro MA, Aoki MS, Coutts AJ, Araújo RC, Bacurau RF. Hormonal, metabolic and perceptual responses to different resistance training systems. J Sports Med Phys Fitness. 2010;50(2):229-34.
16. Burd NA, West DW, Staples AW, Atherton PJ, Baker JM, Moore DR, et al. Low-load high volume resistance exercise stimulates muscle protein synthesis more than high-load low volume resistance exercise in young men. PLoS One. 2010:5(8):e12033.

17. Leite RD, Prestes J, Rosa C, De Salles BF, Maior A, Miranda H, et al. Acute effect of resistance training volume on hormonal responses in trained men. J Sports Med Phys Fitness. 2011;51(2):322-8.

18. Gil S, Roschel H, Batista M, Ugrinowitsch C, TricoliV, Barroso R. Efeito da ordem dos exercícios no número de repetiç̃es e na percepção subjetiva de esforço em homens treinados em força. Rev Bras Educ Fís Esporte. 2011;25(1):127-35.

19. Kuehl RO. Design of experiments: statistical principles of research design and analysis. London: Duxbury-Thomson Learning; 2000.

20. Brown LE, Weir JP. Asep procedures recommendation 1: accurate assessment of muscular strength and power. J Exerc Physiol. 2001;4(3):1-21

21. Borg G. Ratings of perceived exertion and heart rates during short-term cycle exercise and their use in a new cycling strength test. Int J Sports Med. 1982;3(3):153-8.

22. Simão R, Figueiredo T, Leite RD, Jansen A, Willardson JM. Influence of exercise order on repetition performance during low-intensity resistance exercise. Res Sports Med. 2012;20(3-4):263-73.

23. Marshall PW, McEwen M, Robbins DW. Strength and neuromuscular adaptation following one, four, and eight sets of high intensity resistance exercise in trained males. Eur J Appl Physiol. 2011;111(12):3007-16

24. Marshall PW, Robbins DA, Wrightson AW, Siegler JC. Acute neuromuscular and fatigue responses to the rest-pause method. J Sci Med Sport. 2012;15(2):153-8.

25. Ahtiainen JP, Häkkinen K. Strength athletes are capable to produce greater muscle activation and neural fatique during high-intensity resistance exercise than nonathletes. J Strength Cond Res. 2009;23(4):1129-34.

26. Smilios I, Pilianidis T, Karamouzis M, Parlavantzas A, Tokmakidis SP. Hormonal responses after a strength endurance resistance exercise protocol in young and elderly males. Int J Sports Med. 2007;28(5):401-6.

27. Smilios I, Pilianidis T, Karamouzis M, Tokmakidis SP. Hormonal responses after various resistance exercise protocols. Med Sci Sports Exerc. 2003:35(4):644-54.

28. Rønnestad BR, Egeland W, Kvamme NH, Refsnes PE, Kadi F, Raastad T. Dissimilar effects of one- and three-set strength training on strength and muscle mass gains in upper and lower body in untrained subjects. J Strength Cond Res. 2007;21(1):157-63.

29. Hass CJ, Garzarella L, de Hoyos D, Pollock ML. Single versus multiple sets in long-term recreational weightlifters. Med Sci Sports Exerc. 2000;32(1):235-42

30. Wernbom M, Augustsson J, Thomeé R. The influence of frequency, intensity, volume and mode of strength training on whole muscle cross-sectional area in humans. Sports Med. 2007:37(3):225-64. 